\title{
Statistical Birkeland current distributions from magnetic field observations by the Iridium constellation
}

\author{
B. J. Anderson ${ }^{1}$, H. Korth ${ }^{1}$, C. L. Waters ${ }^{2}$, D. L. Green ${ }^{2}$, and P. Stauning ${ }^{3}$ \\ ${ }^{1}$ The Johns Hopkins University, Applied Physics Laboratory, Laurel, MD, USA \\ ${ }^{2}$ School of Mathematical and Physical Sciences, The University of Newcastle, Callaghan, NSW, Australia \\ ${ }^{3}$ Solar-Terrestrial Physics Division, Danish Meteorological Institute, Copenhagen, Denmark
}

Received: 4 December 2006 - Revised: 8 November 2007 - Accepted: 10 December 2007 - Published: 26 March 2008

\begin{abstract}
We present a statistical analysis of Birkeland currents derived from Iridium magnetometer data acquired in the Northern Hemisphere to determine the dependence of large-scale currents on the interplanetary magnetic field (IMF) direction. Because the Iridium data span nearly seven years, we can restrict analysis to only those intervals with stable currents. We used image comparison to quantify the consistency between successive one-hour current distributions and selected 1550 two-hour intervals, $5 \%$ of the data, for analysis. Results include: no statistically significant average currents are present poleward of $80^{\circ}$ during southward IMF; Region-2 currents are weak and confined to latitudes $>65^{\circ}$ during northward IMF; there is marked contrast between currents for northward and southward IMF but the evolution of the patterns is continuous with IMF rotation. The directions of flows inferred from the most poleward currents are more consistent with theoretical expectations of transport away from magnetopause reconnection than previous results. We attribute the differences to the restriction in this analysis to intervals having relatively stable distributions of current so that the data set corresponds more nearly to pure states of the system.
\end{abstract}

Keywords. Ionosphere (Electric fields and currents) - Magnetosphere (Current systems; Magnetosphere-ionosphere interactions)

\section{Introduction}

Birkeland currents play a central role in the transport of energy and momentum to the ionosphere by conveying stress between the magnetosphere and the ionosphere. They are a fundamental signature of the solar wind-magnetosphereionosphere (M-I) interaction and their distribution reflects

Correspondence to: B. J. Anderson

(brian.anderson@jhuapl.edu) the dynamic state of the system. The M-I system and hence the distribution and intensity of the large-scale Birkeland currents is largely governed by the magnitude and direction of the interplanetary magnetic field (IMF), the solar wind speed and density, and the ionospheric conductivity. Characterizing these dependencies is central to understanding driven MI electrodynamics. Previous studies have determined global distributions of the large-scale Birkeland currents and their relationship to solar wind and IMF parameters by statistical analysis of single-satellite observations (Iijima and Potemra, 1978; Weimer, 2001, 2005; Papitashvili et al., 2002). These studies significantly advanced our understanding of M-I coupling and may represent the most comprehensive analyses possible with these data. From this body of work we know that the IMF orientation is the dominant factor controlling the distribution of the currents and that while the IMF magnitude and the solar wind speed and density affect the intensity of the currents, they do not to first order alter the pattern of the currents.

More recent studies are not consistent with respect to the configuration of currents at high latitudes, $>75^{\circ}$ MLAT. In some cases, currents persist at high latitudes for all IMF orientations. This is particularly evident in the Papitashvili et al. (2002) analysis which is based on the high precision MAGSAT and Ørsted data. In these results, the NBZ currents (e.g. Iijima et al., 1984; Zanetti et al., 1984) have comparable intensities for purely northward and southward IMF. This persistence of the NBZ currents is evident to a lesser degree, in the Weimer (2001) results. The persistent NBZ system is difficult to reconcile with statistical analyses of the electric field observations from DE-2 (Heppner and Maynard, 1987; Weimer, 1995, 1996) and plasma drift measurements from DMSP (Papitashvili and Rich, 2002), which detect strong convection associated with northward IMF and NBZ currents but find only anti-sunward convection over the polar cap for southward IMF. A persistent NBZ system has so far not been evident during southward IMF conditions in

Published by Copernicus Publications on behalf of the European Geosciences Union. 
single event analyses of two dimensional Birkeland currents derived from Iridium magnetometer data (e.g. Waters et al., 2001; Korth et al., 2004). If persistent polar cap currents really are present, this would have significant implications for our concept of convection and energy deposition in the polar ionosphere.

Conversely, in the Weimer (2005) analysis the NBZ currents are not present for southward IMF but for northward IMF they are weaker than the equatorward Region-1 sense currents. In addition, the Region 2 currents are as strong or stronger than the NBZ currents. This would suggest that the dominant flows are not associated with the NBZ filamentary currents but with the persistent strong Region-1 sense currents. Since the reconnection driven anti-sunward flows are not expected under these conditions, this would imply that the viscous interaction is as strong or stronger than northward IMF reconnection responsible for the NBZ currents. This is not consistent with the case study of Korth et al. (2005) and the simulations of the same event (Merkin et al., 2007), showing that the NBZ currents are stronger than the Region-1 sense currents while the Region 2 currents are barely detectable. These conundrums and inconsistencies between different studies for both northward and southward IMF motivate the additional analysis of Birkeland current dependence on the IMF orientation presented here.

Global distributions of the Birkeland currents spanning February 1999 to the present have been derived from magnetic field data returned by the constellation of Iridium satellites. The Iridium constellation consists of over 70 satellites in six 780-km circular, near-polar orbits. As part of the avionics, each satellite carries a magnetometer that is sensitive enough to detect perturbations from the Birkeland currents (Anderson et al., 2000). Due to the large size of the engineering telemetry block containing the magnetic field samples, the telemetry rate of these data to the ground is rather coarse, about one sample from each satellite every $200 \mathrm{~s}$. Nonetheless, these data allow derivation of global Birkeland current distributions on one-hour time scales using the technique described by Waters et al. (2001). The latitude resolution obtained with a data accumulation of one hour is approximately $4^{\circ}$. The Iridium global fits of the magnetic perturbations have been verified to be reliable by comparison with in-situ observations by the Ørsted and DMSP satellites provided that the Iridium magnetic field sampling is everywhere finer than the shortest latitude resolved and that the perturbations are consistent over the accumulation interval (Korth et al., 2004, 2005; Waters et al., 2004).

The Iridium data set is well suited to determine a statistical description of the Birkeland currents. First, because the Iridium system yields a two-dimensional distribution every hour, these data can be used to identify intervals for which the currents are stable. By testing the current distributions of sequential intervals for stability, one can impose a quantitative restriction that the M-I system remained in a consistent state during the interval in question. Second, using only stable intervals ensures that the solar wind and IMF observations recorded by the Advanced Composition Explorer (ACE) at L1 accurately reflect the conditions at Earth. In the analysis presented here, the intervals used are one hour long, longer than the uncertainties in the delay between L1 and Earth. Third, the data set is large so one can afford to select a small fraction of the data for analysis and still have enough data to yield statistically significant results.

This paper presents results of statistical analysis of 1550 stable two-hour intervals identified in Northern Hemisphere observations from almost seven years of observations, to determine the dependence of the currents on the IMF orientation. We focus on the morphology of the current patterns emphasizing differences relative to prior analyses. The key findings are: the absence of statistically significant average high latitude currents for southward IMF; the minimal Region-2 currents for northward IMF; and the smooth, continuous evolution of the current patterns as the IMF rotates from northward to southward consistent with expectations for flows associated with magnetopause reconnection. The data analysis is described in Sect. 2, and the statistical distributions are presented in Sect. 3 and discussed in Sect. 4.

\section{Data analysis}

The Iridium data used for this study span nearly seven years, from February 1999 through December 2005, with greater than $97 \%$ coverage. The derivation of magnetic perturbations from the raw data and evaluation of the Birkeland currents from the perturbations are described by Anderson et al. (2000) and Waters et al. (2001), respectively. The present analysis uses Northern Hemisphere data because the current distributions from Iridium are generally more accurate there than in the south. Because of the greater eccentricity of the magnetic pole in the south, the Iridium constellation orbit crossing point (near the geographic pole) more often falls within the regions of large-scale currents in the south than in the north. Where this happens the fit results tend to be less reliable owing both to the fact that the input data are crosstrack perturbations only and to singularities in the spherical harmonic fit near the orbit crossing point. Techniques to circumvent these present limitations are under development, but are not required to address the science issues of the present study, so we focus exclusively on the Northern Hemisphere data. To identify intervals suitable for analysis, we calculated Birkeland current distributions for every hour of observations available and compared distributions for sequential hours to determine the degree to which the current pattern was stable. We then quantitatively examined the degree to which this data subset represents more stable IMF than average using data from the ACE spacecraft located at L1. Data from the magnetometer (Smith et al., 1998) and the solar wind plasma instrument (McComas et al., 1998) were lagged in time using simple advection from L1 to the Earth's center using the 
proton bulk speed. The timing uncertainty of this approach is approximately 10-20 $\mathrm{min}$, shorter than the one-hour intervals used for Iridium data. The average solar wind and IMF for each one-hour interval of Iridium data were calculated and used to bin the events by IMF direction.

\subsection{Stable current event identification}

Stability of the currents was measured as follows. The data set yielded just over 60000 one-hour Birkeland current distributions so we used an automated comparison method. Object identification techniques commonly used in image analysis (Haralick et al., 1987) were applied to every sequential pair of current distributions to calculate the average percentage overlap of the upward and downward current regions having the strongest currents. For each pair of distributions, denoted 1 and 2, we identified the peak upward current density in distribution 1 and expanded the region around this extremum to include all current densities greater than the $2 \sigma$ confidence level (Korth et al., 2004). The area of this region is denoted $A_{1}$. Next we identified all upward current regions in distribution 2 , denoted by the index $i$, and calculated the areas of each of these regions, $A_{2, i}$, also above the $2 \sigma$ confidence level. We then calculated the area of overlap between $A_{1}$ and the $i$ th region in distribution 2, written as $A_{\mathrm{o} 12, i}$. The fractional overlap was then evaluated from

$R_{\mathrm{o} 12, i}=A_{\mathrm{o} 12, i} /\left(A_{1}+A_{2, i}-A_{\mathrm{o} 12, i}\right)$

The denominator is the area of the union of $A_{1}$ and $A_{2, i}$. We identified the largest $R_{\mathrm{O} 12, i}$, denoted $R_{\mathrm{O} 12 \text {,max }}$, and saved $R_{\mathrm{o} 12, \text { max }}$ as well as the area of overlap, $A_{\mathrm{o} 12 \text {,max }}$. The calculation was repeated for downward current and then also with distributions 1 and 2 interchanged, that is, by first finding the maximum current densities in distribution 2 . The mean of the resulting four maximum overlap fractions, written $\bar{R}_{\mathrm{o} 12 \text {, max }}$, measures the average overlap between the two distributions.

Figure 1 illustrates the results of this analysis for four examples corresponding to southward (a), northward (b), dawnward (c), and duskward (d) IMF orientations. Upward and downward currents are shown in red and blue, respectively. Gray shading indicates current densities below the $2 \sigma$ confidence level (Korth et al., 2004). The overlaid solid traces are the overlap regions. In the left hand panels the contours are from comparison of the earlier interval with the latter (forward), and in the right hand panels they are from comparison of the latter with the earlier (reverse). Table 1 gives the $\bar{R}_{\mathrm{O} 12, \max }$ values for each pair together with the advection time lagged average solar wind and IMF quantities for each one-hour interval.

The examples illustrate how the algorithm identifies the large-scale currents most in common between the patterns, regardless of the form of the distribution. Except for the downward currents in Fig. 1b, the current density extrema occurred in the same regions in both intervals so the forward and reverse overlap regions are identical. The contours in a) $10 / 19 / 2001$ 18:00 - 19:00 UT
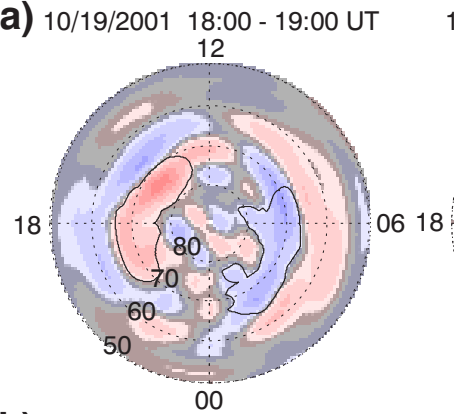

10/19/2001 19:00 - 20:00 UT

b) $05 / 07 / 200010: 00$ - 11:00 UT

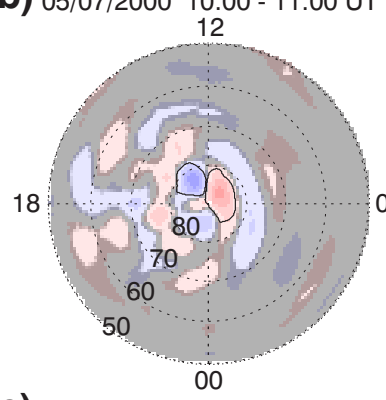

C) $03 / 23 / 200215: 00-16: 00$ UT
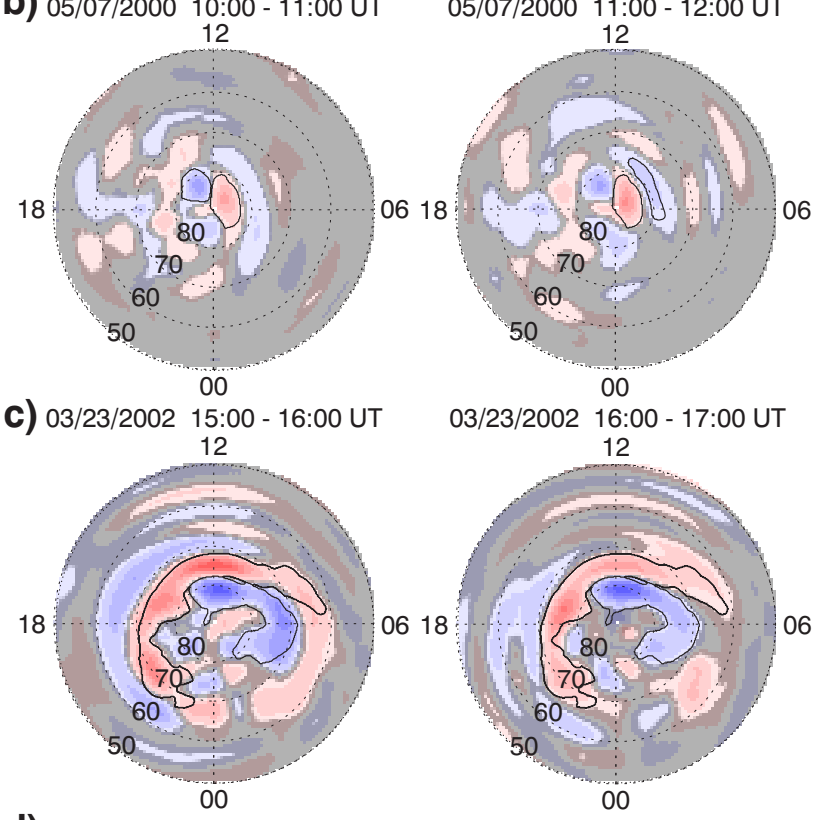

d)
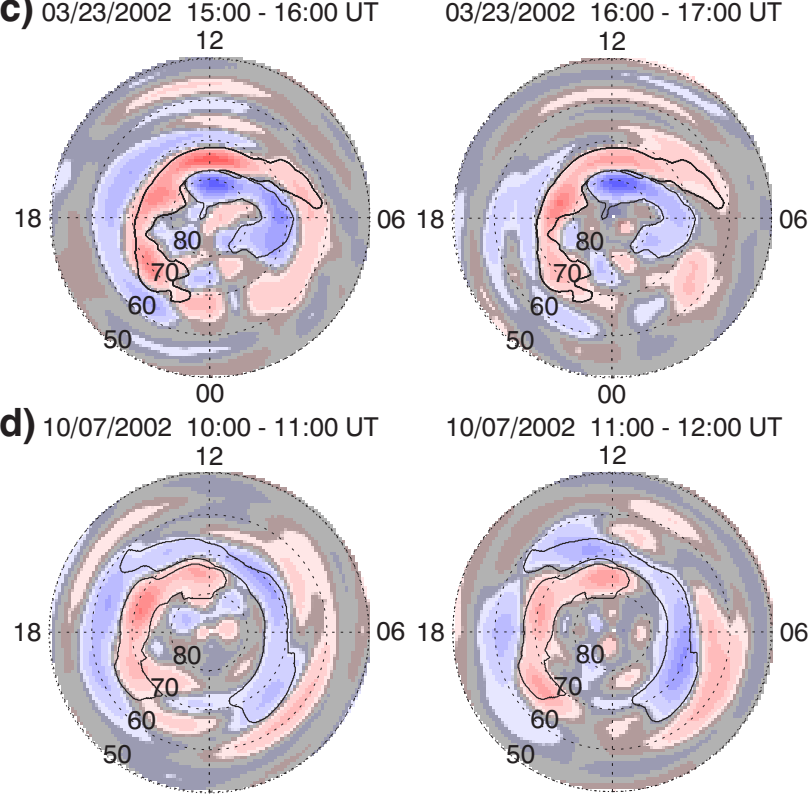

10/07/2002 11:00 - 12:00 UT

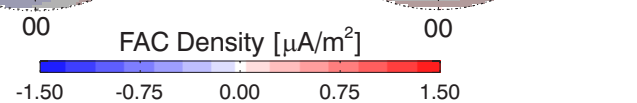

Fig. 1. Example Birkeland current distributions for (a) southward, (b) northward, (c) dawnward, and (d) duskward IMF orientation. Upward (downward) currents are in red (blue) and gray shading indicates currents below the $2 \sigma$ confidence level. Contours in the left hand panels show the overlap region identified by finding the region with maximum current density (upward or downward) in the earlier interval and evaluating the overlap of this region above the $2 \sigma$ level with the later interval. Contours on the right show the overlap found by finding the region with maximum current density first in the later interval and evaluating the overlap with the earlier interval. 
Table 1. Current region overlap fraction and average solar wind and IMF parameters for the intervals of Fig. 1. All ACE data are lagged using simple advection. The clock angle is evaluated from the average vector projection in the $Y$ - $Z$ GSM plane.

\begin{tabular}{|c|c|c|c|c|c|c|c|c|}
\hline \multirow[t]{2}{*}{ Event: Date } & \multirow[t]{2}{*}{$\bar{R}_{\mathrm{O} 12, \max }$} & \multirow{2}{*}{$\begin{array}{l}\text { Time } \\
\text { (UT) }\end{array}$} & \multirow{2}{*}{$\begin{array}{l}\text { IMF Clock } \\
\text { Angle }\left(^{\circ}\right)\end{array}$} & \multicolumn{3}{|c|}{$\mathrm{IMF}(\mathrm{nT})$} & \multirow{2}{*}{$\begin{array}{c}n_{\mathrm{p}} \\
\left(\mathrm{cm}^{-3}\right)\end{array}$} & \multirow{2}{*}{$\begin{array}{c}v_{\mathrm{p}} \\
(\mathrm{km} / \mathrm{s})\end{array}$} \\
\hline & & & & $B_{\mathrm{X}}$ & $B_{\mathrm{y}}$ & $B_{\mathrm{Z}}$ & & \\
\hline a: 19 Oct 2001 & 0.62 & 18:00-19:00 & 170 & -3.8 & 1.3, & -7.0 & 6.8 & 341 \\
\hline$"$ & - & 19:00-20:00 & 153 & -5.4 & 3.2 , & -6.3 & 6.3 & 337 \\
\hline b: 7 May 2000 & 0.74 & 10:00-11:00 & 14 & 3.4 & 1.6 & 6.7 & 8.1 & 403 \\
\hline & - & 11:00-12:00 & 16 & 4.5 & 1.7 & 5.9 & 7.8 & 399 \\
\hline c: 23 March 2002 & 0.56 & $15: 00-16: 00$ & -110 & 2.9 & -8.8 & -3.2 & 11.7 & 461 \\
\hline$"$ & - & $16: 00-17: 00$ & -114 & 2.4 & -8.6 & -3.8 & 12.9 & 456 \\
\hline d: 10 July 2002 & 0.50 & 10:00-11:00 & 103 & -5.5 & 8.0, & -1.9 & 10.3 & 394 \\
\hline$"$ & - & $11: 00-12: 00$ & 117 & -4.3 & 7.4, & -3.7 & 11.2 & 420 \\
\hline
\end{tabular}

Fig. 1b for negative current are different because the maximum negative current density occurred in different regions for the earlier and later intervals. The algorithm therefore identified different regions to use in evaluating the overlap. In all other cases the maximum magnitude current densities occurred in overlapping regions so the comparison algorithm picked the same contours in the forward and reverse comparisons. Even though the automated comparison primarily identifies the more poleward large-scale currents, e.g. Region 1 in Fig. 1a, the correspondence in large-scale currents is evident for the lower latitude currents as well. The set of events span the range of current distributions from southward IMF (Iijima and Potemra, 1976) to NBZ currents for northward IMF (Iijima et al., 1984; Zanetti et al., 1984) and symmetrically distorted patterns for IMF $B_{\mathrm{y}}$ dominated conditions of opposite sign (Potemra et al., 1984; Cowley et al., 1991). The image comparison algorithm successfully picks consistent pairs of distributions over the full range of patterns that were found to occur.

Examination of many examples indicated that a threshold value of $\bar{R}_{\mathrm{o} 12, \max }=0.45$ identified those pairs of distributions that one would visually identify as consistent. The total number of distribution pairs with $\bar{R}_{\mathrm{O} 12 \text {, } \max }>0.45$ was 1550 representing $3100 \mathrm{~h}$ or $\sim 5 \%$ of the total observations. These stable distributions are the basis of our statistical analysis.

\subsection{Assessment of IMF stability}

The primary difference between our work and prior studies is that we restrict analysis to a subset of the data for which the current patterns were relatively stable. In our selection process, the events were selected solely according to the stability of the Birkeland current pattern without regard to IMF or solar wind variability. While one would expect that stable currents should occur for solar wind/IMF conditions that are also stable, particularly with respect to IMF direction, it is important to check if this is the case. We therefore examined the degree of IMF direction stability for every two-hour interval and compared populations with greater and lesser sta- bility in the Birkeland currents to see if they correspond to different degrees of IMF stability.

We quantified IMF directional stability as follows. The unit vector of each four-minute averaged IMF magnetic field value was constructed, denoted $\mathbf{n}_{\mathrm{B}, i}$. The mean unit vector was then calculated and renormalized to yield a unit vector, $\mathbf{n}_{\mathrm{B}, \mathrm{Avg}}$, corresponding to the average IMF direction. The angular departure of each $\mathbf{n}_{\mathrm{B}, i}$ from $\mathbf{n}_{\mathrm{B} \text {, Avg }}$ is

$\delta_{\mathrm{B}, i}=\cos ^{-1}\left(\mathbf{n}_{\mathrm{B}, i} \cdot \mathbf{n}_{\mathrm{B}, \mathrm{Avg}}\right)$

From the $\delta_{\mathrm{B}, i}$ we calculated the average and maximum, $\delta_{\mathrm{Avg}}$ and $\delta_{\text {Max }}$, for each two-hour interval. Note that the maximum $\delta_{\mathrm{Avg}}$ and $\delta_{\mathrm{Max}}$ are $90^{\circ}$ and $180^{\circ}$, respectively. Because the X GSM component of the IMF does not contribute to the solar wind electric field to first order, we also calculated analogous quantities using only the projection of the IMF in the $Y-Z$ GSM plane. That is, from the $Y$ and $Z$ components we constructed the $Y-Z$ plane unit vectors, $\mathbf{n}_{\mathrm{BYZ}, i}$, the renor-

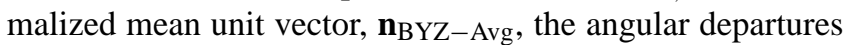
from the mean $Y-Z$ plane unit vector, $\delta_{\mathrm{BYZ}, i}$, and finally the average and maximum angular departures in the $Y-Z$ plane from the mean $Y-Z$ plane direction, $\delta_{\mathrm{YZ}-\mathrm{Avg}}$ and $\delta_{\mathrm{YZ}-\mathrm{Max}}$. In the following we simply use " $\delta$ statistics" or just " $\delta$ " when referring to the set, $\delta_{\mathrm{Avg}}, \delta_{\mathrm{Max}}, \delta_{\mathrm{YZ}-\mathrm{Avg}}$, and $\delta_{\mathrm{YZ}-\mathrm{Max}}$.

Figure 2 shows the occurrence distributions of the $\delta$ statistics for the entire data set of two-hour intervals. These distributions are normalized by the maximum occurrence number so that the quantities shown are the ratios of the probability of occurrence, $P$, to the maximum probability, $P_{\text {Max }}$. The maxima in the $\delta_{\mathrm{Avg}}$ and $\delta_{\mathrm{YZ}-\mathrm{Avg}}$ distributions are $90^{\circ}$ for the simple reason that the average departure from the mean direction in a nearly random directional distribution is $90^{\circ}$. The largest values of $\delta_{\text {Max }}$ and $\delta \mathrm{YZ}-$ Max are obviously $180^{\circ}$. All of the distributions have deep minima near $0^{\circ}$, reflecting the fact that the IMF direction is almost always varying. The IMF direction over two-hour intervals is most likely to vary by $\sim 20^{\circ}$ on average from the mean direction though the most likely $Y-Z$ plane departure from the mean is smaller, $10^{\circ}$ to 


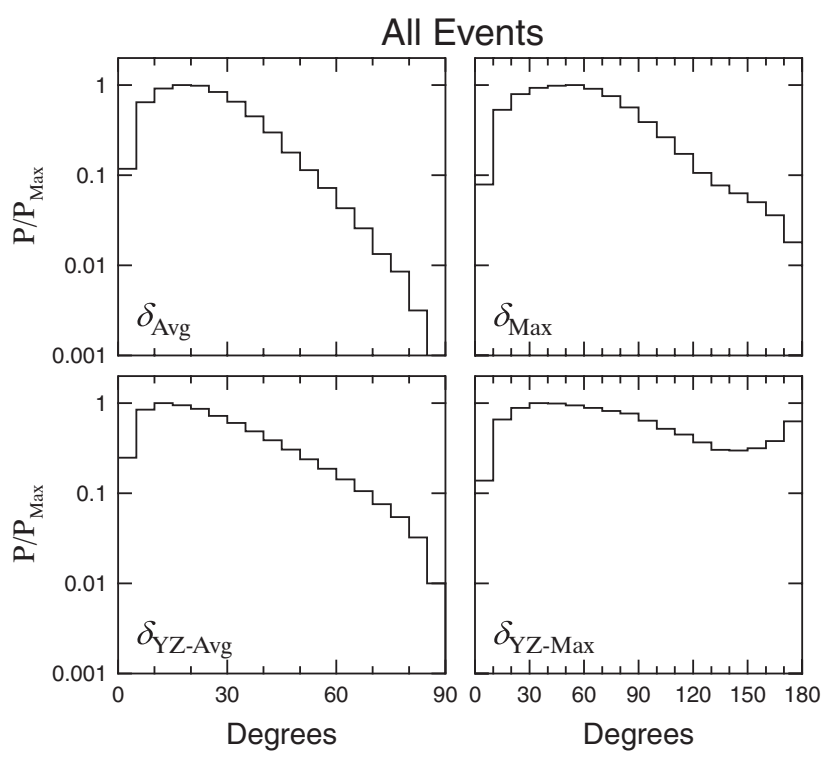

Fig. 2. Occurrence distributions of IMF direction variation statistics over the entire set of two-hour intervals having Iridium-derived Birkeland currents from February 1999 through 2005. All distributions are normalized by the maximum occurrence number of each distribution. The angle $\delta$ is the departure from the mean IMF direction in the two-hour interval. The subscripts Avg, Max and YZ indicate the average departure, the maximum departure and directions evaluated only in the $Y-Z$ GSM plane projection, respectively.

$15^{\circ}$. The shapes of the 3-D and $Y-Z$ plane $P / P_{\text {Max }}$ distributions are similar though the 3-D distribution falls off more rapidly as $\delta_{\mathrm{Avg}}$ increases. A $\delta_{\mathrm{Avg}}$ of $\sim 60^{\circ}$ occurs about ten times less often than the most likely $\delta_{\mathrm{Avg}}$.

The $P / P_{\text {Max }}$ distributions for $\delta_{\mathrm{Max}}$ and $\delta_{\mathrm{YZ}-\mathrm{Max}}$ are more uniform, particularly in the $Y-Z$ plane. The $\delta_{\mathrm{YZ}-\mathrm{Max}}$ distribution rises near $180^{\circ}$ owing to the fact that if the IMF $\mathrm{X}$-component dominates, a small rotation of the IMF in 3$\mathrm{D}$ can lead to a large rotation of its $Y-Z$ plane projection. The most probable $\delta_{\mathrm{Max}}$ is in the range $25^{\circ}$ to $40^{\circ}$ with a $\delta_{\text {Max }}$ of $120^{\circ}$ being about ten times less common. For $\delta_{\mathrm{YZ}-\text { Max }}$ the most probable value is about $40^{\circ}$ but the minimum at higher $\delta$ YZ-Max never falls below about 0.3 reflecting a greater range of variation in the $Y-Z$ plane projection.

We now consider whether the set of events with relatively stable current patterns represents more stable than average IMF directions, that is, whether they are weighted toward low $\delta$ statistics. We take subsets of events in three overlap percentage ranges, $0-20 \%, 45-60 \%$, and $60-100 \%$. For each subset we normalize the $\delta$ distributions by dividing them by the occurrence rates of all events so that the value for each $\delta$ bin is the fraction of events represented by the subset having $\delta$ in that range. There were $\sim 14000$ intervals in the $0-20 \%$ overlap subset, 1478 in the $45-60 \%$ subset, and 91 in the 60 $100 \%$ subset. To compare the occurrence distributions for

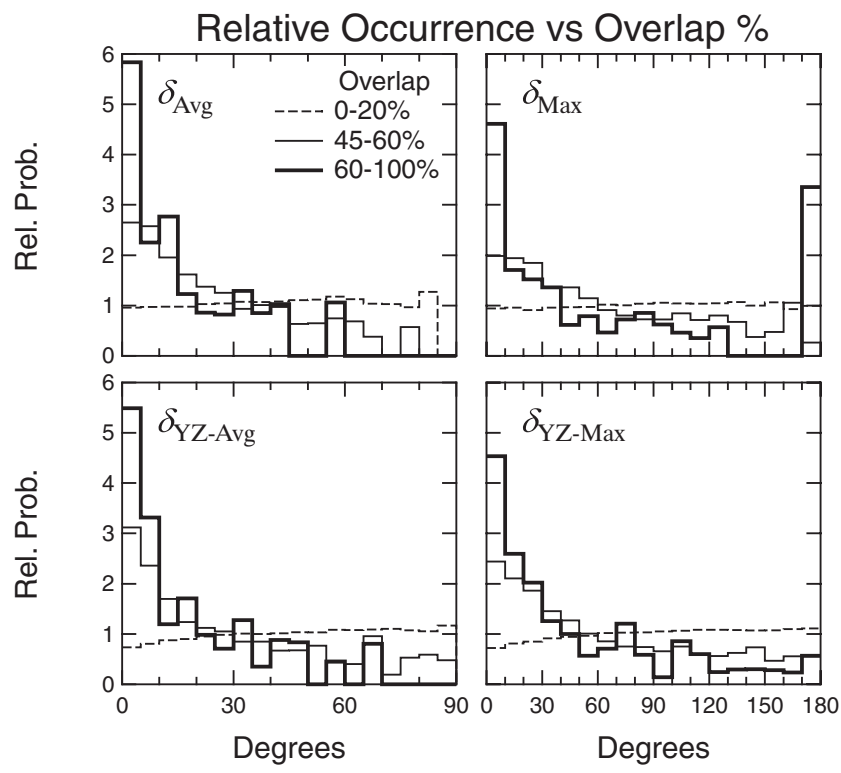

Fig. 3. Relative occurrence probabilities of average and maximum angular departure from average IMF orientation in two-hour intervals for events in three Birkeland current overlap ranges: 0-20\% (dashed lines), 45-60\% (solid thin lines), and 60-100\% (solid thick lines).

subsets of these vastly different sample sizes, the fractional distributions were re-scaled so that average value was unity. These normalized, re-scaled distributions are called relative probability distributions. A relative probability distribution that had the same distribution in $\delta$ as the entire data set would have a value of 1 for all $\delta$.

Figure 3 shows the relative probability distributions for $\delta_{\mathrm{Avg}}, \delta_{\mathrm{YZ}-\mathrm{Avg}}, \delta_{\mathrm{Max}}$, and $\delta_{\mathrm{YZ}-\mathrm{Max}}$ for all three subsets of data. The $0-20 \%$ overlap subset is shown by the dashed lines, the $45-60 \%$ subset by the thin lines and the $60-100 \%$ subset by the thick lines. It is immediately clear that all of the distributions for the $45-60 \%$ and $60-100 \%$ overlap subsets are skewed toward low $\delta$, with the relative probability of both being greater than 1 for $\delta_{\text {Avg }}$ less than about $30^{\circ}$ and for $\delta_{\text {Max }}$ below about $50^{\circ}$ or $60^{\circ}$. The relative probabilities of the 45 $60 \%$ and $60-100 \%$ subsets increase with decreasing $\delta$ and exceed 2 for any $\delta$ below $10^{\circ}$. The probability of an event with a $45-60 \%$ or $60-100 \%$ overlap decreases with increasing $\delta$. The scatter at high $\delta_{\text {Max }}$ is due to the low number of events in the bins for $\delta_{\text {Max }}$ near $180^{\circ}$. In particular, there are very few events in the $170^{\circ}-180^{\circ}$ bin for $\delta_{\mathrm{Max}}$, and the value in the $\delta_{\text {Max }}$ distribution for the $60-100 \%$ subset represents a single event.

The relative probability distributions for $0-20 \%$ overlap in the Birkeland current patterns are markedly different. The distributions are nearly independent of $\delta$ being only slightly below 1 for $\delta_{\mathrm{Avg}}$ below $30^{\circ}$ and $\delta_{\mathrm{Max}}$ below $60^{\circ}$, and slightly above 1 for higher $\delta$. The distributions $\delta_{\mathrm{YZ}-\mathrm{Avg}}$ and $\delta_{\mathrm{YZ}-\mathrm{Max}}$ show a drop in relative probability for low $\delta$, indicating that 


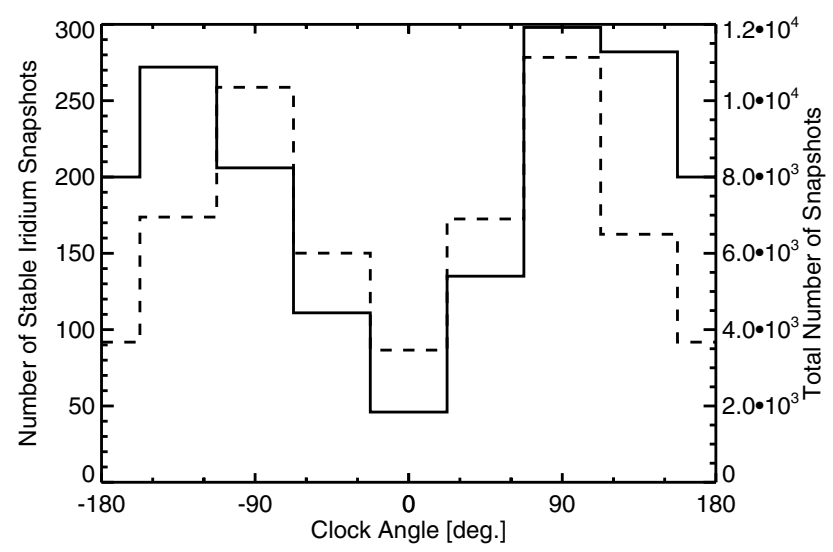

Fig. 4. Comparison of the IMF clock angle distribution from 1999 to 2005 (dashed line, right hand scale) with that of the 1550 stable two-hour Iridium events used for this study (solid line, left hand scale).

the low overlap fraction events become less likely when the IMF is stable in the $Y-Z$ plane. This analysis demonstrates that the events selected here based on the overlap percentage in their Birkeland current distributions do correspond to conditions that are more likely to be stable in the IMF direction. Moreover, as the degree of overlap increases, the bias toward stable IMF (low $\delta$ ) also increases. Thus, with respect to previous studies that did not select events for IMF stability, our population is biased toward conditions with stable IMF direction.

Although not of direct interest for purposes of this paper, it is interesting to note that stable IMF direction does not guarantee that events pass our stable currents test. Nor does a stable current pattern necessarily imply that the IMF direction was stable. We identify several observational and physical reasons why a stable IMF direction does not necessarily mean that the Birkeland current patterns as recorded by Iridium will be stable. First, a stable IMF is not sufficient to ensure that currents will be strong relative to the noise level in our analysis. Currents in local winter are known to be relatively weak and most of our events come from northern summer months. In addition, the data density in the Iridium data may adversely affect the overlap analysis, since if the distribution of data samples is not comparable from one hour to the next, the derived current patterns may be sufficiently different to fail our criteria. Lastly, even though the IMF is stable, the currents may not be stable owing to internal magnetospheric dynamics.

The results also indicate why currents could be included in our stable pattern subset even though the IMF direction is variable. It is apparent that the $45 \%$ lower limit in overlap allows for some variation in the currents. That the $60-100 \%$ overlap distribution is significantly more strongly biased toward small $\delta$ suggests that the $45-60 \%$ subset, representing the vast majority of the events selected for analysis, includes a number of events for which the currents really did change but not so much as to reduce the overlap below our $45 \%$ lower limit. The paucity of events in the $60-100 \%$ range may reflect the fact that the M-I system rarely remains truly stable for as long as two hours. Thus even though our "stable" current pattern events represent more stable IMF than the average variability and hence are more selective than prior analyses, there remains some degree of real variation in the currents in the data set we have selected.

\subsection{Stable event average solar wind and IMF properties}

To organize the stable current events according to IMF direction, we calculated the average solar wind and IMF properties for each event. The clock angle, $\psi$, was calculated as the arctangent of the $Y-Z$ GSM projection of the average IMF, $\psi=\arctan \left(B_{\mathrm{y}}, B_{\mathrm{z}}\right)$, such that positive $\psi$ indicates duskward IMF, $\psi=0^{\circ}$ is northward, and $|\psi|=180^{\circ}$ is southward. The events were then binned by $\psi$ in $45^{\circ}$ wide bins with bin centers at $\psi=0^{\circ}, \pm 45^{\circ}, \pm 90^{\circ}, \pm 135^{\circ}$, and $|\psi|=180^{\circ}$. Figure 4 compares the $\psi$-distribution of our events (solid line) with that for the entire interval from 1999 through 2005 (dashed line). While both distributions peak for $|\psi|$ near $90^{\circ}$, the distribution of all data has comparable minima for $\psi=0^{\circ}$ and $|\psi|=180^{\circ}$, whereas the stable event $\psi$-distribution has more events near $|\psi|=180^{\circ}$ than near $\psi=0^{\circ}$. Nonetheless, the stable events span the entire range of $\psi$ with good statistics in each bin; there are 46 events in the $\psi=0^{\circ}$ bin and at least 100 events in all other bins.

Although not critical for our analysis, it is useful to consider the reason for the southward IMF bias in the event distribution. One possibility is that the solar wind driving must be stronger to produce more intense currents for northward than for southward IMF. To check this, we evaluated the average solar wind electric field projected into the $Y-Z$ GSM plane, i.e. $E_{\mathrm{yz}}=\left|v_{\mathrm{x}} \sqrt{B_{\mathrm{y}}^{2}+B_{\mathrm{z}}^{2}}\right|$, for each $\psi$-bin for the entire data interval and for the stable events. For all data there was no dependence of $E_{\mathrm{yz}}$ on $\psi$ as expected and the average $E_{\mathrm{yz}}$ was $2.05 \mathrm{mV} / \mathrm{m}$. While the event set also showed no significant variation of the average $E_{\mathrm{yz}}$ with $\psi$, the average $E_{\mathrm{yz}}$ for our stable events was $2.7 \mathrm{mV} / \mathrm{m}$ indicating that the events correspond to stronger solar wind driving than average. Our event selection is therefore biased somewhat toward stronger driving than average and hence most likely to more intense currents. Given that Birkeland currents are stronger in the summer months (Papitashvili et al., 2002), the bias in the event selection toward strong currents may imply a seasonal dependence of the selection process.

There is in fact a significant seasonal effect in event selection. The ratio of the number of events within three months of northern summer solstice to those within three months of winter solstice was 2.6:1. The seasonal influence in turn favors southward IMF conditions in our event selection. This 


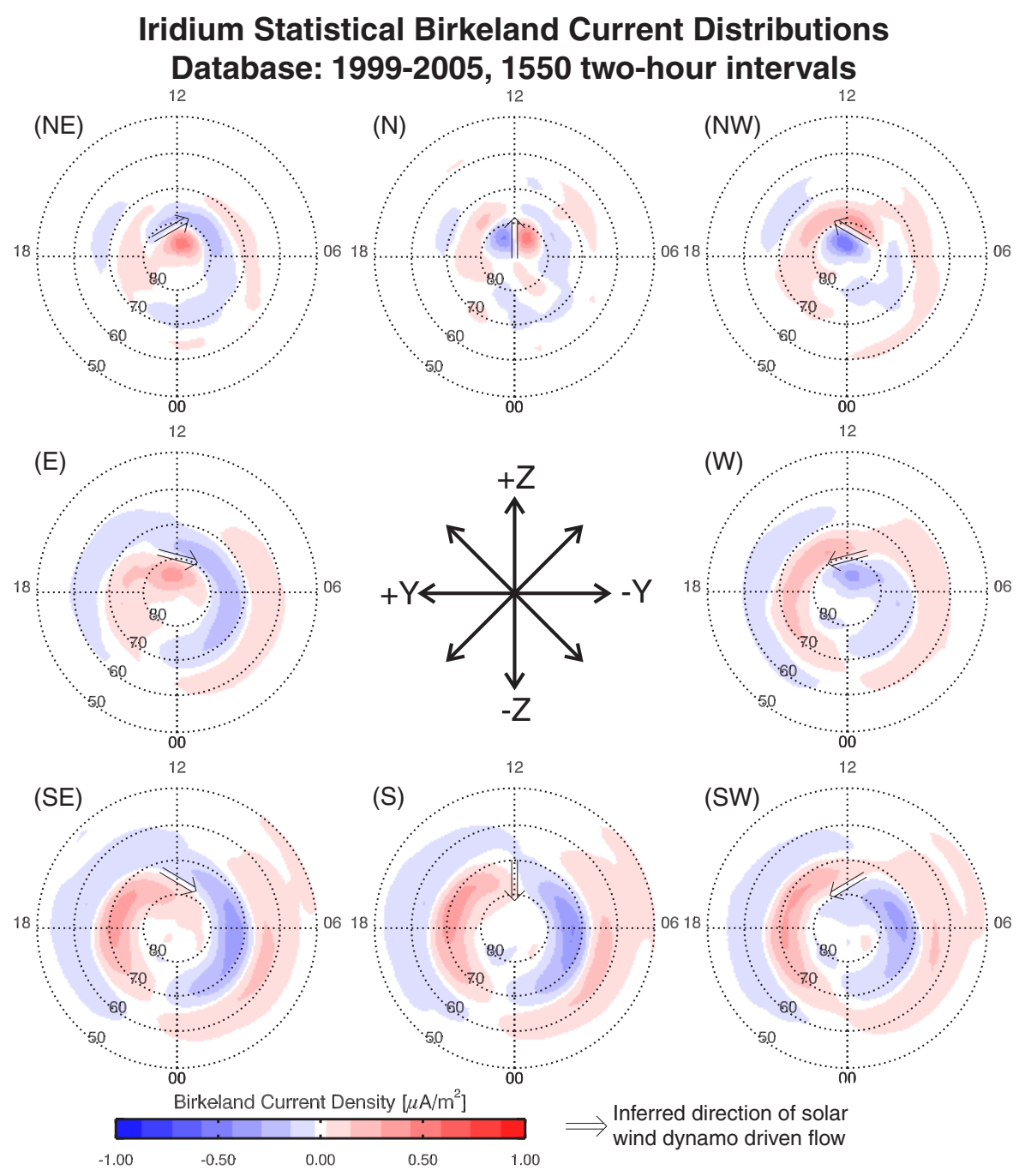

Fig. 5. Statistical distributions of the large-scale Birkeland currents obtained from analysis of 1550 two-hour global distributions observed by Iridium. The IMF direction projected in the $Y-Z$ GSM plane for each distribution is indicated by the arrows in the center and by the panel labels corresponding to the IMF direction at the subsolar magnetopause. The $2 \sigma$ uncertainty levels are given in the lower right corner of each panel. Double line arrows show the inferred direction of flow implied by the most poleward currents.

is confirmed in the seasonal distribution for different $\psi$-bins; for the $\psi=0^{\circ}$ bin, the summer/winter event ratio is 3.6:1 whereas for the $|\psi|=180^{\circ}$ bin the ratio is $2.5: 1$. Since the currents are at lower latitudes for southward than northward IMF and since the EUV conductance is on average higher where the southward IMF currents close, the currents should be stronger for southward than northward IMF conditions for comparable dipole tilts. We therefore attribute the lower number of northward IMF cases in our event $\psi$-distribution relative to the IMF distribution as reflecting the seasonal dependence of current intensity.

\section{Statistical Birkeland currents}

Statistical distributions of the currents were obtained by averaging the distributions within each clock angle bin. The results are shown in Fig. 5 using the same red (up) and blue (down) color scheme and polar format as Fig. 1 but without gray shading. Statistics of the current distributions for each IMF clock angle bin are given in Table 2. In the figure, the color to white transition approximates the $2 \sigma$ standard error in the mean given in the table, and which does not vary greatly with clock angle, so that statistically insignificant currents are below the color level. The distributions are ordered by IMF orientation as indicated by the arrows in the figure's center panel. The notations $+Z$ and $+Y$ correspond to IMF northward $(\mathrm{N})$ and duskward or eastward (E) at the subsolar 
dayside magnetopause, respectively. The panels are labeled indicating the bin center clock angle, e.g. (SW) for southwest, $-Z$ and $-Y$. The black double line arrows overlaid on each distribution indicate the direction of plasma flow inferred from the most poleward pair of large-scale currents. Table 2 gives the number of two-hour intervals in each averaged distribution, the minimum (i.e. downward) and maximum (upward) current density in each averaged distribution, the average $2 \sigma$ uncertainty in the current density, the corresponding $2 \sigma$ error in the mean, and the total current, $I_{\text {total }}$. The total current was obtained by integrating the current in each region above the $2 \sigma$ standard error in the mean, adding up the absolute values of these integrated currents and dividing by two. The uncertainty listed for $I_{\text {total }}$ is half of the difference in magnitude of the integrated upward and downward current.

The evolution of the currents is well ordered by IMF clock angle. For southward IMF, panel (S), the Iridium averages show the Region-1 and Region-2 currents discussed by Iijima and Potemra (1976); Region-1 currents are downward (upward) on the dawnside (duskside) with the opposite for Region-2 currents. Our results show that the axis of symmetry for southward IMF is tilted slightly toward pre-midnight (pre-noon) relative to the noon-midnight meridian consistent with average patterns of ionospheric convection (Weimer, 1995, 1996; Papitashvili and Rich, 2002; Ruohoniemi and Greenwald, 1996, 2005). Southward IMF corresponds to a total current of $1.82 \mathrm{MA}$, a factor of 5.5 greater than for northward IMF, 0.33 MA. In contrast, the maximum average current densities do not vary as dramatically with clock angle. The totals of the average current distributions are useful for comparison between the averages even though they may not accurately reflect the total current of individual distributions which are typically higher (e.g. Korth et al., 2004, 2005).

The current distributions evolve progressively as the IMF turns away from southward consistent with the influence of the IMF $B_{\mathrm{y}}$ (Potemra et al., 1984; Cowley et al., 1991). For southward and duskward (SE) (dawnward (SW)) IMF, the duskside upward (dawnside downward) Region-1 current curls poleward near noon while the opposite polarity Region1 current merges continuously across noon with the duskside (dawnside) Region-2 current. The total current remains comparable to that for southward IMF and the maximum upward and downward current densities are similar.

As the IMF rotates so that $B_{\mathrm{y}}$ dominates, (E) and (W), the basic topology is preserved but the areas of the currents decrease and move poleward and the total current deceases to a bit more than half the southward IMF value. In addition, the most poleward Region-1 sense current, upward for (E) and downward for (W), has maximum intensity at noon just sunward of the pole. These Region-1 sense currents near the magnetic pole are the regions of most intense current density, upward (positive) for $\psi=90^{\circ}(\mathrm{E})$ and downward (negative) for $\psi=-90^{\circ}$. For these IMF $B_{\mathrm{y}}$ dominated conditions it is not clear whether the most poleward current should be termed Region 1 or Region 0. Although it appears to have evolved from the southward IMF Region 1 system, these currents are often termed Region 0 (e.g. Ohtani et al., 1995). We therefore use the descriptive term "Region-1 sense" to indicate that the current has the same sense as the corresponding Region 1 for southward IMF from which it appears to have evolved. From the inferred flows we argue below that their relationship to the solar wind dynamo driven reconnection flows are the same as the southward IMF Region-1 currents.

The topology of the currents changes as the IMF turns northward. When $B_{\mathrm{y}}$ and $B_{\mathrm{z}}$ are comparable, (NE) and (NW), the Region-2 currents are markedly weakened relative to strong $B_{\mathrm{y}}$ or southward IMF conditions. The total current is nearly halved again relative to the (E) and (W) IMF conditions. However, the most poleward current at noon, up for (NE) and down for (NW), is more intense than for (E) and (W) and this small region of current appears to be losing connection with the corresponding Region-1 sense current at dusk for (NE) or dawn for (NW). The maximum current density in this filament near the pole is slightly higher than the maximum for more southward $\psi$. In addition, the opposite Region-1 sense current, down for (NE) and up for (NW), is losing continuity with the same sense Region- 2 current. For (NE) the dusk equatorward most Region-2 sense current is completely isolated and the dawn equatorward most Region2 sense current for (NW) is only barely contiguous with the Region- 1 sense of the same sign.

For northward IMF $(\mathrm{N})$, the NBZ currents near the pole are the most prominent feature and the extreme current densities are both higher than for southward IMF. Owing to their small area the total current is a minimum. The most poleward currents for (NW) and (NE) are now side by side forming the NBZ currents that bound the sunward convection flow (e.g. Eriksson et al., 2005; Korth et al., 2005). The Region-1 sense currents of complementary polarity are located just equatorward of the NBZ system. These Region-1 sense currents at dusk (up) and dawn (down) are now completely separated from the localized currents associated with the NBZ currents. The Region- 2 currents are faint remnants of their southward IMF counterparts and are confined to the dayside in small areas poleward of about $65^{\circ}$.

The contrast between northward and southward IMF bears more quantitative comparison. The integrated Region2 sense currents for southward IMF were 0.87 MA and $-0.63 \mathrm{MA}$, where we denote upward as positive and downward as negative. By contrast, for northward IMF they were $0.06 \mathrm{MA}$ and $-0.03 \mathrm{MA}$, indicating that the decrement in total Region-2 sense current is a factor of 10 to 20. The Region-1 sense currents for southward IMF were $1.06 \mathrm{MA}$ and $-1.07 \mathrm{MA}$ whereas for northward IMF they were $0.16 \mathrm{MA}$ and $-0.12 \mathrm{MA}$. These are nearly identical to the NBZ total currents which are $0.14 \mathrm{MA}$ and $-0.12 \mathrm{MA}$. If the magnitude of the residual Region- 2 current reflects the effects of a viscous interaction, these results indicate that even 
Table 2. Statistics of the averaged current distributions for each clock angle bin.

\begin{tabular}{|c|c|c|c|c|c|c|c|}
\hline \multirow{2}{*}{$\begin{array}{l}\text { Clock } \\
\text { Angle }\end{array}$} & & \multirow{2}{*}{$\begin{array}{r}\text { No. } \\
\text { Events }\end{array}$} & \multicolumn{4}{|c|}{$j\left(\mu \mathrm{A} / \mathrm{m}^{2}\right)$} & \multirow{2}{*}{$\begin{array}{l}I_{\text {total }} \\
\text { (MA) }\end{array}$} \\
\hline & & & Min & Max & $\operatorname{Avg} 2 \sigma$ & $\operatorname{Std-Err}(2 \sigma)$ & \\
\hline-180 & S & 200 & -0.44 & 0.41 & 0.19 & 0.013 & $1.82 \pm 0.12$ \\
\hline-135 & SW & 272 & -0.37 & 0.32 & 0.17 & 0.010 & $1.56 \pm 0.12$ \\
\hline-90 & W & 206 & -0.35 & 0.30 & 0.14 & 0.010 & $0.95 \pm 0.15$ \\
\hline-45 & NW & 111 & -0.58 & 0.39 & 0.12 & 0.011 & $0.58 \pm 0.16$ \\
\hline 0 & $\mathrm{~N}$ & 46 & -0.47 & 0.58 & 0.12 & 0.018 & $0.33 \pm 0.04$ \\
\hline 45 & $\mathrm{NE}$ & 135 & -0.27 & 0.54 & 0.12 & 0.010 & $0.50 \pm 0.10$ \\
\hline 90 & $\mathrm{E}$ & 298 & -0.26 & 0.36 & 0.15 & 0.008 & $1.02 \pm 0.09$ \\
\hline 135 & SE & 282 & -0.39 & 0.38 & 0.18 & 0.011 & $1.83 \pm 0.04$ \\
\hline
\end{tabular}

for northward IMF it contributes not more than about $30 \%$ of the current. If the viscous contribution is comparable for northward and southward IMF, then the relative contribution for southward IMF is $\sim 6 \%$ of the Region- 2 current.

The relationship of the Iridium results to M-I system dynamics can be illustrated by considering the flows implied by the currents. The double line arrows in Fig. 5 illustrate the flow directions implied by the most poleward currents. The strongest ionospheric electric field is approximately directed from the peak downward current to the peak upward current and the flow direction given by $\boldsymbol{E} \times \boldsymbol{B}$, where $\boldsymbol{B}$ is downward. The inferred most poleward flows are anti-sunward for southward IMF and sunward for northward IMF. For positive (negative) IMF $B_{\mathrm{y}}$, the flows are directed toward dawn (dusk) and rotate progressively from anti-sunward to sunward as the IMF turns northward. These flows are in agreement with theoretical and simulation results for convection away from magnetopause reconnection (e.g. Cowley et al., 1991). The present results therefore indicate a direct correspondence between the Birkeland currents and solar wind dynamo forcing and in particular that the most poleward currents are intimately linked with magnetopause reconnection, regardless of the IMF orientation.

\section{Relationship to previous analyses}

The differences between these results and previous statistical models by Weimer (2001), Papitashvili et al. (2002), and Weimer (2005) are most evident in the contrast between northward and southward IMF conditions. For southward IMF the Iridium distributions do not show significant currents poleward of $80^{\circ}$ MLAT whereas some previous results show statistically significant NBZ-sense currents for all IMF orientations. From Fig. 3 we estimate that the upper limit for these currents in our result is approximately $30 \%$ of the northward IMF NBZ intensities. For northward IMF, the Region-1 and particularly the Region-2 currents, are reduced in area and intensity compared to previous models. Alternatively, Weimer (2005) indicate that the NBZ currents are weaker than the Region-1 sense currents for northward IMF and that Region 2 current densities are comparable to those of the NBZ system, whereas we find that the NBZ current densities are considerably greater than the Region-1 sense currents. We also find that the Region 2 currents are mostly restricted to the dayside for northward IMF. Even though the difference between northward and southward IMF current distributions is greater in our results the previous studies, the evolution of the currents between these extremes is clear and systematic indicating a regular transformation of the M-I system.

To identify reasons for these differences and determine which results are more reliable, we first need to check whether high precision magnetic field data are consistent with our statistical results. To do this, we identified events in the $|\psi|=180^{\circ}$ and $\psi=0^{\circ}$ bins of our stable currents data base for which Ørsted vector magnetometer data were available. From these we picked cases having tracks of the Ørsted satellite that passed through both the NBZ currents and/or the large-scale Region 1 and 2 currents. To compare with the Iridium results we evaluated the horizontal magnetic perturbations from both our statistical result and the Iridium fit for each event along the Ørsted track and transformed the statistical and fit results into along and cross track components in the Ørsted frame. Four cases are shown below in Figs. 6 through 9 and the solar wind/IMF conditions for these events are given in Table 3 .

For the $\psi=0^{\circ}$ bin there were twenty cases with at least one Ørsted pass with vector magnetometer data. For many of these, the Ørsted track did not pass through the NBZ system and the Ørsted data did not show perturbations larger than the baseline residuals. Figure 6 shows data from 19 August 2003 in which Ørsted passed nearly through the center of the NBZ system from 12:21 to 13:06 UT. The Iridium data from 11:00 to 13:00 UT correspond to one of the events in our stable current data base. The IMF was northward with $B_{\mathrm{Z}}>13$ nT (cf. Table 3).

The figure shows the detrended Iridium cross-track magnetic field perturbations as red arrows in panel (a) together 
Table 3. Current region overlap fraction and average solar wind and IMF parameters for the Ørsted comparison intervals of Figs. 6, 7, 8, and 9 as indicated in the Event: date column and in order from top to bottom. All ACE data are lagged using simple advection. The clock angle is evaluated from the average vector projection in the $Y-Z$ GSM plane.

\begin{tabular}{|c|c|c|c|c|c|c|c|c|}
\hline \multirow[t]{2}{*}{ Event: Date } & \multirow[t]{2}{*}{$\bar{R}_{\mathrm{O} 12, \max }$} & \multirow{2}{*}{$\begin{array}{l}\text { Time } \\
\text { (UT) }\end{array}$} & \multirow{2}{*}{$\begin{array}{l}\text { IMF Clock } \\
\text { Angle }\left(^{\circ}\right)\end{array}$} & \multicolumn{3}{|c|}{$\mathrm{IMF}(\mathrm{nT})$} & \multirow{2}{*}{$\begin{array}{c}n_{\mathrm{p}} \\
\left(\mathrm{cm}^{-3}\right)\end{array}$} & \multirow{2}{*}{$\begin{array}{c}v_{\mathrm{p}} \\
(\mathrm{km} / \mathrm{s})\end{array}$} \\
\hline & & & & $B_{\mathrm{X}}$, & $B_{\mathrm{y}}$, & $B_{\mathrm{Z}}$ & & \\
\hline 4: 19 Aug 2003 & 0.58 & $11: 00-12: 00$ & -3 & -2.8 & -1.9 & 14.5 & 6.8 & 453 \\
\hline 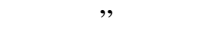 & - & $12: 00-13: 00$ & 12 & -0.5 & 2.9 & 13.7 & 5.0 & 445 \\
\hline 5: 4 Aug 2002 & 0.46 & $13: 00-14: 00$ & -5 & -0.4 & -0.6 & 7.0 & 6.7 & 455 \\
\hline & - & $14: 00-15: 00$ & -25 & -0.8 & -3.4 & 6.9 & 6.3 & 462 \\
\hline 6: 4 Oct 2002 & 0.51 & $16: 00-17: 00$ & -160 & -0.9 & -2.5 & -7.0 & 4.8 & 413 \\
\hline$"$ & - & 17:00-18:00 & -166 & -2.1 , & -1.7 & -6.9 & 4.7 & 401 \\
\hline 7: 1 Oct 2002 & 0.57 & $11: 00-12: 00$ & 158 & -4.2 & 8.8 & -21.7 & 15.0 & 413 \\
\hline$"$ & - & $12: 00-13: 00$ & 160 & -4.0 & 7.7, & -21.0 & 14.7 & 410 \\
\hline
\end{tabular}

with the Ørsted horizontal magnetic perturbation as black arrows starting at the Ørsted position when the data were recorded. We used one second Ørsted data for the analysis but the plot shows one Ørsted value every 20 s for clarity. Panel (b) shows the Iridium Birkeland current distribution with the Ørsted magnetic perturbations overlaid in the same fashion as panel (a). The line plots in (c) show: cross-track (upper) and along-track (lower) Ørsted data with solid black traces; Iridium magnetic perturbation fit evaluated along the Ørsted track with dashed black traces; and magnetic perturbations for the statistical $\psi=0^{\circ}$ average multiplied by two with the red traces. Since we are primarily interested in assessing whether the average result locates the magnetic perturbations in the same regions as observed by Ørsted, the $\psi=0^{\circ}$ result was multiplied by two, solely to make it easier to see. We attach no significance to the factor of two and it is not a quantitative measure of the underestimation in the statistical result.

A word of caution is warranted regarding currents derived from Iridium for individual cases such as this event. In examining individual current distributions derived from Iridium data, the reliable currents are only those associated with the largest magnetic perturbations. Because the fitting process is only constrained by the cross-track magnetic perturbation, the fit will occasionally produce currents that are consistent with the cross-track data but which imply predominantly along-track perturbations at the locations of the observations. Hence, although consistent with the input data, the fit process can yield low level "phantom" currents for which there is no compelling evidence in the actual input data. Since these spurious currents are consistent with the observations and arise solely because the fit is not ideally constrained by the data, they are often above the two-sigma uncertainty estimate of Korth et al. (2004). We therefore guard against misinterpreting or over-interpreting the individual event distributions by emphasizing that currents in regions where the cross-track perturbations are essentially at the noise level are not to be trusted and should be ignored in individual events. Consistent with this caution, for both the 19 August 2003 and 4 August 2002 events (Figs. 6 and 7), only the NBZ currents near noon on the dayside were clearly consistent between the two one-hour distributions of each event.

The comparison in Fig. 6 shows that the locations and sense of the perturbations from both the individual Iridium event analysis and the Iridium statistical average are consistent with the Ørsted observations. Considering panel (a), the locations of the enhanced dawnward cross-track perturbations at about $78^{\circ}$ MLAT in the early afternoon as well as the cross-track reversal at about $83^{\circ}$ MLAT are reflected in both the Iridium and Ørsted data. It is the Iridium data on the track just pre-noon where the cross-track perturbations were duskward in the comparable dayside latitude range, that establish the twin vortex nature of the perturbation field indicative of the NBZ system. From panel (b) it is clear that the Ørsted data reflect primarily the signatures of the strong downward current of the NBZ pair since Ørsted passed just to the dusk side of the center between the upward and downward currents. The Region-1 sense currents were not detected by Ørsted on this pass.

The line plots show that the Iridium fits (dashed traces) for this event locate the maxima and reversals in both the cross and along track components quite well while underestimating the actual maxima by a factor of two to three. The Ørsted NBZ signatures for this case exceed $400 \mathrm{nT}$ whereas the extrema in the Iridium fit are $200 \mathrm{nT}$ in amplitude. This underestimation in the maxima is consistent with the fairly low latitude resolution of $\sim 4^{\circ}$ that that the Iridium data density supports. Even though the fit underestimates the intensity, the structure of the large scale features is captured in the Iridium result. Despite considerable attenuation, the statistical average also locates the largest amplitude features in agreement with the large-scale perturbations from Ørsted.

Figure 7 shows results for a $\psi=0^{\circ}$ case with a dawndusk Ørsted track tor comparison with the $|\psi|=180^{\circ}$ results 


\section{8/19/2003 11:00 - 13:00 UT (North)}
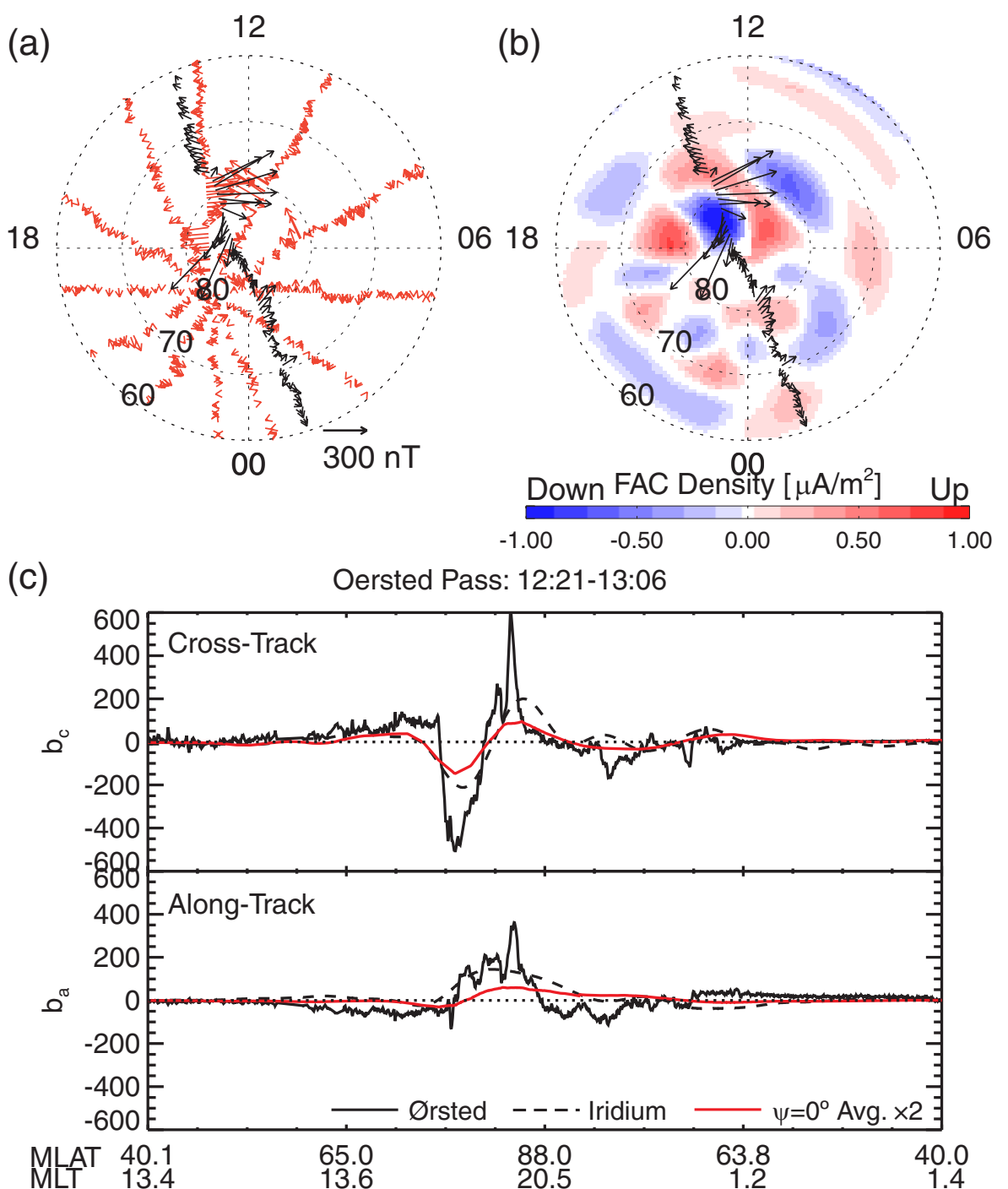

Fig. 6. Ørsted and Iridium observations for a northward IMF event from 19 August 2003. Iridium data are from 11:00 to 13:00 UT and Ørsted data are from the Northern Hemisphere pass from 12:21 to 13:06 UT. Figures show comparison of Ørsted horizontal magnetic field perturbations (black arrows and solid black traces) with (a) Iridium cross-track perturbations (red arrows), (b) Birkeland currents derived from Iridium data (red-blue color scale), and (c) Ørsted data (black trace), spherical harmonic fit vector horizontal field from Iridium (dashed trace), and twice the $\psi=0^{\circ}$ statistical average (red line) evaluated along the Ørsted track. Ørsted data in panels (a) and (b) are shown as arrows starting at the Ørsted location. Panel (c) shows the horizontal components in cross, $b_{c}$, (upper panel) and along, $b_{a}$, (lower panel) track directions. Average solar wind and IMF conditions for this interval are given in Table 3.

considered below, for which dawn-dusk Ørsted tracks are the most definitive. Actually, this is the only $\psi=0^{\circ}$ case with a dawn-dusk Ørsted track for which the vector data are available. For this event, the magnetic perturbations are smaller, reaching only $200 \mathrm{nT}$ or so in both the Ørsted and Iridium data. Despite this, the Iridium data show the same basic features as Ørsted, namely, anti-sunward perturbations near noon with sunward perturbations on both the dusk and dawn sides of the noon-midnight meridian, all of which indicate the filamentary NBZ currents. This signature in the crosstrack component is evident in Ørsted as well as the Iridium event and statistics. The along-track signature is reflected better in the Iridium event analysis than in the statistical fit, but the basic large-scale structures are consistent with Ørsted. From panel (b) we see that Ørsted passed just to the nightside of the current system and the rotational signatures in the Ørsted data agree with the Iridium-derived Birkeland currents. 


\section{8/04/2002 13:00 - 15:00 UT (North)}
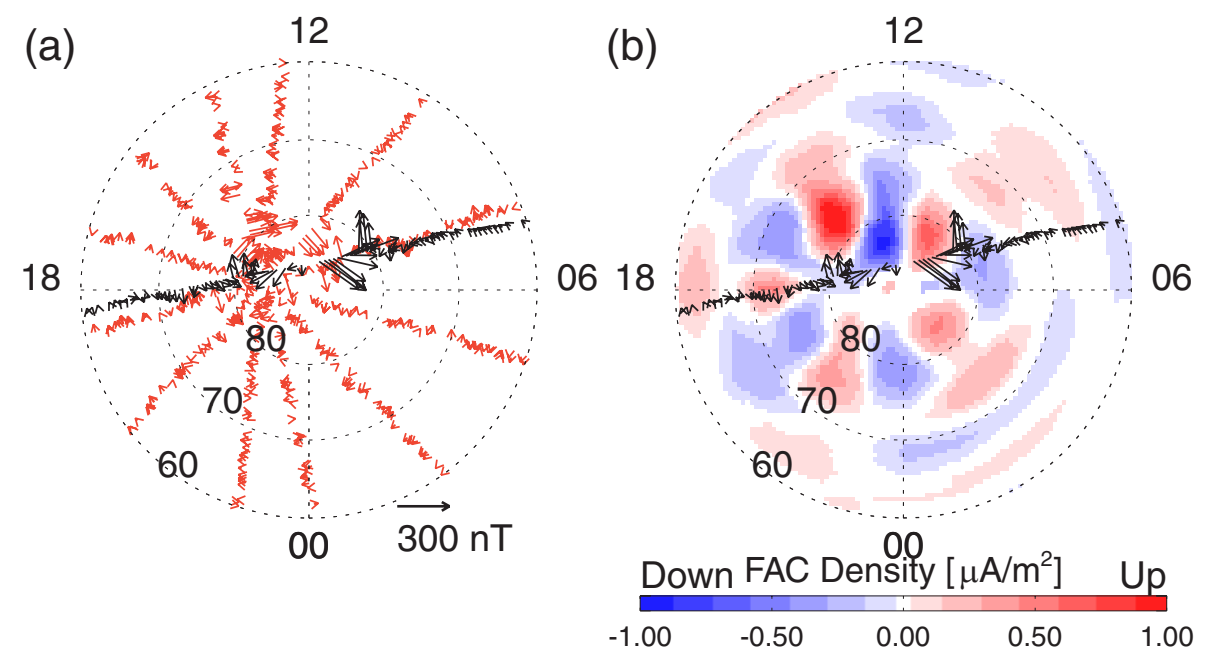

(c)

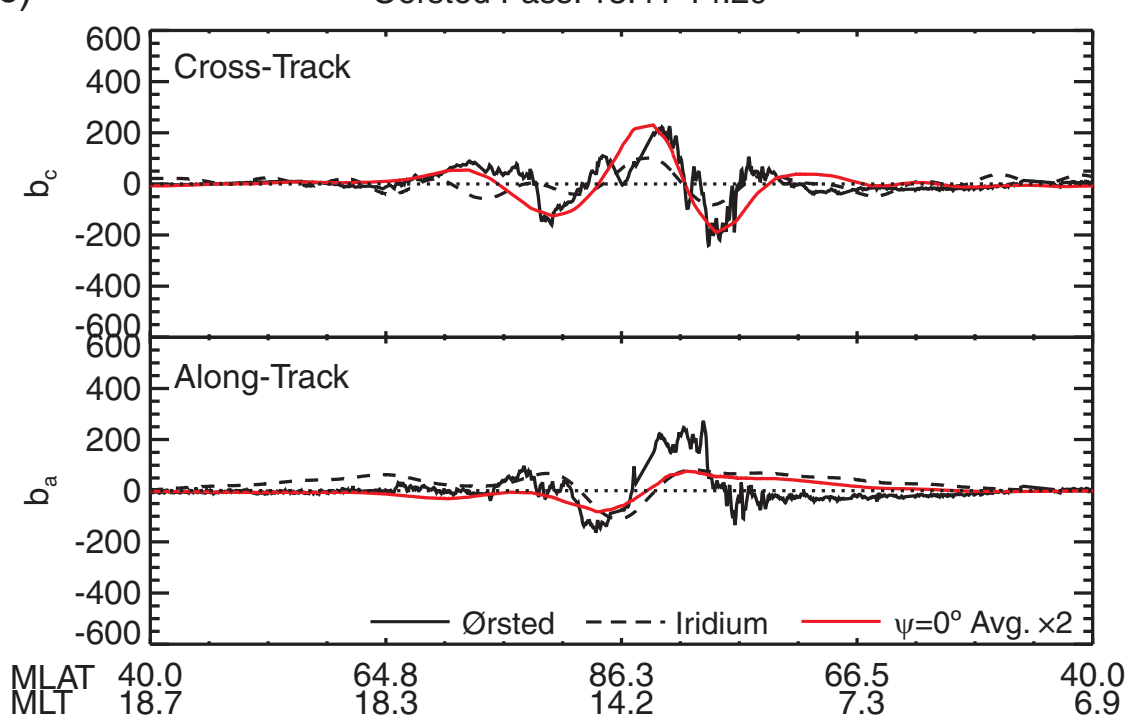

Fig. 7. Ørsted and Iridium observations for a northward IMF event from 4 August 2002 in the same format as Fig. 6. Iridium data are from 13:00 to 15:00 UT and Ørsted data are from the Northern Hemisphere pass from 13:41 to 14:29 UT. Average solar wind and IMF conditions for this interval are given in Table 3.

Figure 8 shows the comparison with Ørsted data for a southward IMF interval on 4 October 2002 (cf. Table 3). In this case Ørsted tracks dusk to dawn just to the night side of the dawn-dusk meridian and the data show prominent Region 1 and 2 current signatures. The Iridium fit is in close agreement with Ørsted at dusk. At dawn the agreement is also good though the Iridium fit places the dawn maximum perturbation slightly equatorward of that observed by Ørsted. The Iridium fit does not capture the along-track (north-south) perturbations at dawn very well. Both Iridium and Ørsted indicate prevailing sunward perturbations over the polar cap. Significantly, the Ørsted data indicate an absence of structured currents in the polar cap. There is a gradual rota- tion from slightly dawnward to duskward as Ørsted traverses from dusk to dawn. For comparison with the NBZ current signatures, the red traces in panel (c) show the perturbations corresponding to the $\psi=0^{\circ}$ statistical average evaluated along the Ørsted track, again multiplied by two. This comparison shows that even though Ørsted passed to the night side, it would have detected perturbations of an NBZ-like system, had they been present, consistent with the northward IMF statistical result.

The last example from 1 October 2002, shown in Fig. 9, is also for southward IMF but for much stronger driving, cf. Table 3. Both the Ørsted and Iridium perturbations are quite large for this event, exceeding $1000 \mathrm{nT}$, consistent with 


\section{0/04/2002 16:00 - 18:00 UT (North)}

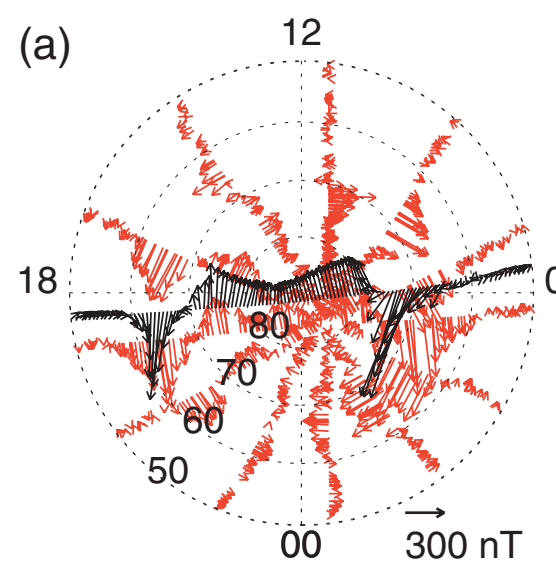

(b)

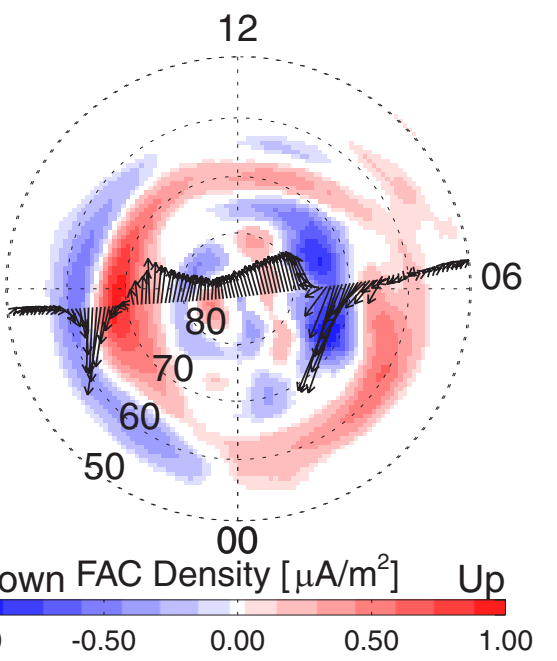

(c)

Oersted Pass: 16:05-16:53

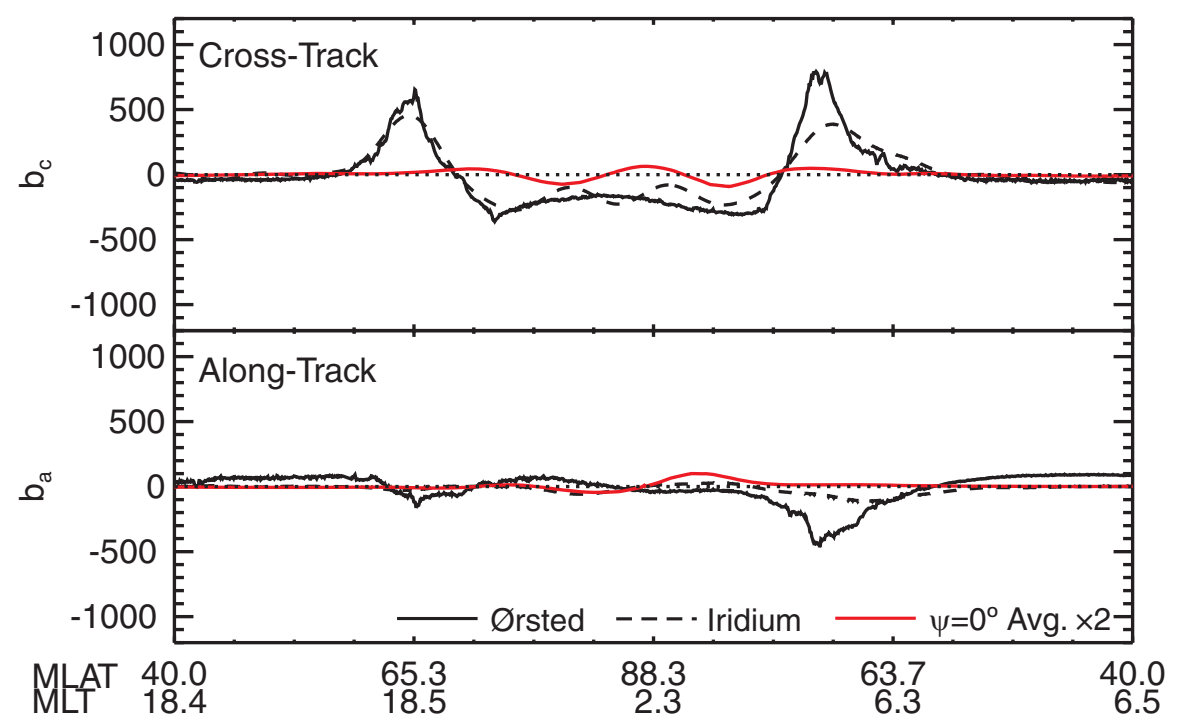

Fig. 8. Ørsted and Iridium observations for a southward IMF event from 4 October 2002 in the same format as Fig. 6. Iridium data are from 16:00 to 18:00 UT and Ørsted data are from the Northern Hemisphere pass from 16:05 to 16:53 UT. The red traces in panel (c) are the $\psi=0$ Iridium statistical result evaluated along the Ørsted track multiplied by two and are shown to indicate the signatures that would have been anticipated for NBZ-like polar cap currents. Average solar wind and IMF conditions for this interval are given in Table 3.

the strong IMF, $B_{\mathrm{Z}}<-21 \mathrm{nT}$. The Iridium fit closely matches the cross-track Ørsted data and locates the large scale currents in close agreement with Ørsted even though the fit does not capture the along track signals recorded by Ørsted. The Ørsted track for this case passes near the center location of the northward IMF NBZ system. While there is a rotation in the Ørsted data poleward of $80^{\circ}$ MLAT in the afternoon, the Ørsted data do not have signatures consistent with the statistical NBZ perturbations shown in red. We note that the polar cap signatures in the 1 October 2002 and 4 October 2002 southward IMF cases both correspond to a rotation from dawnward pointing to duskward pointing indicative of signatures from distant currents rather than passage through a set of currents at high latitudes.

These examples reflect what we find generally in examining cases from our stable currents data base for which Ørsted data are available. The high precision magnetic field data are consistent with the Iridium results in the sense that the same large-scale currents are indicated by both. While the Iridium statistical result is significantly attenuated relative to both Ørsted and the Iridium event fit, the location and structure of the currents are consistent with Ørsted. For northward IMF 


\section{0/01/2002 11:00 - 13:00 UT (North)}

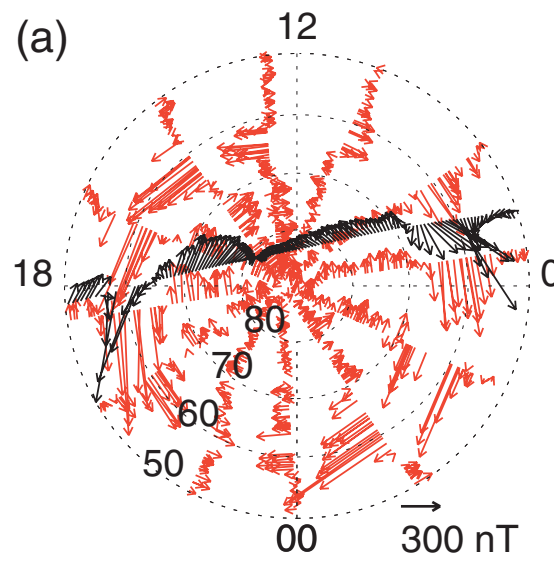

(b)

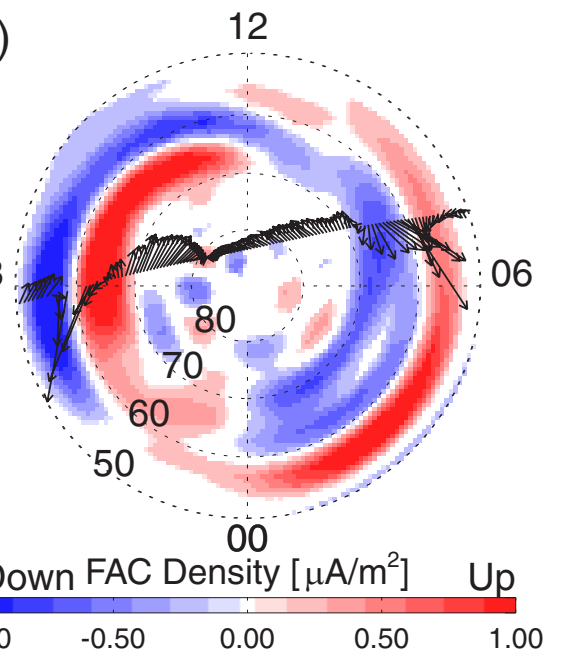

(c)

Oersted Pass: 11:37-12:27

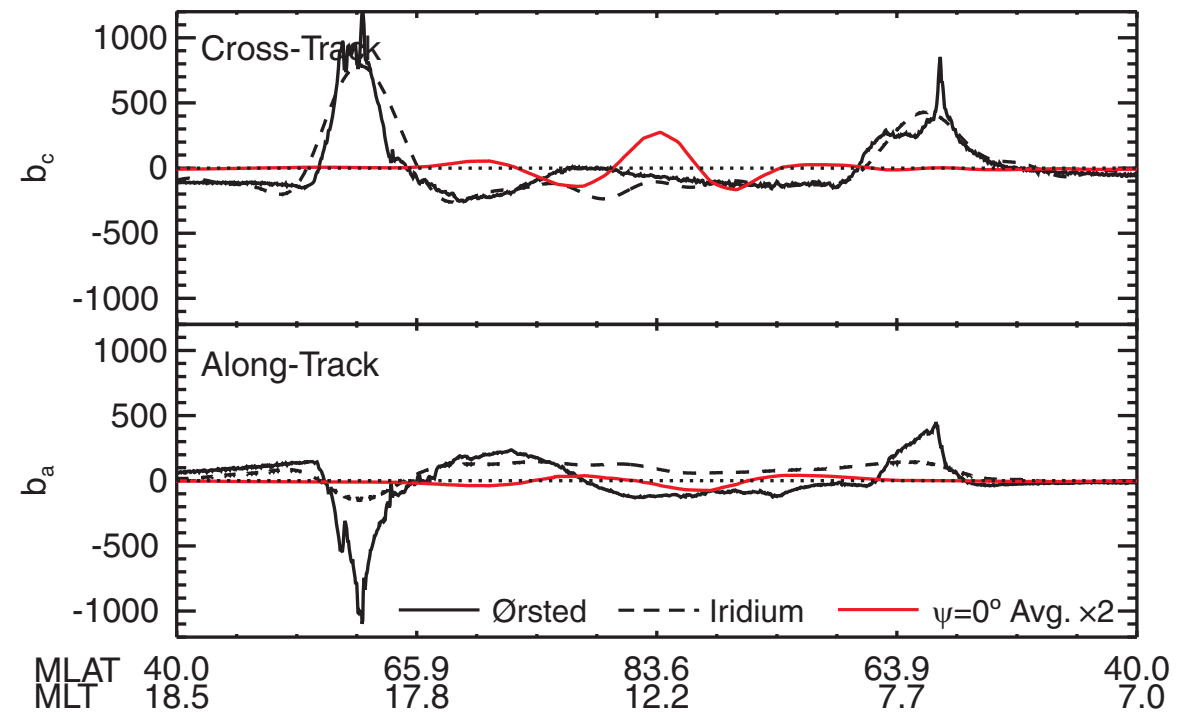

Fig. 9. Ørsted and Iridium observations for a southward IMF event from 1 October 2002 in the same format as Fig. 6. Iridium data are from 11:00 to 13:00 UT and Ørsted data are from the Northern Hemisphere pass from 11:37 to 12:27 UT. The red traces in panel (c) are the $\psi=0$ Iridium statistical result evaluated along the Ørsted track multiplied by two and are shown to indicate the signatures that would have been anticipated for NBZ-like polar cap currents. Average solar wind and IMF conditions for this interval are given in Table 3.

we find that the Ørsted data do not show evidence for a strong Region 2 system, consistent with the statistical Iridium data. Similarly, for southward IMF, the Ørsted data do not indicate persistent polar cap, NBZ-like, currents, also consistent with the Iridium statistics. Finally, the Ørsted data confirm the relative intensity of the NBZ filaments and the Region 1 currents reflected in the Iridium statistics. The differences between our results and prior analyses therefore cannot be attributed to greater sensitivity of the instrumentation on which the other studies were based. Some other factor must be responsible.
We suggest that the present results differ from statistical studies using data from single satellites because previous studies did not constrain the input data for stability of the observed currents whereas the Iridium data afford the luxury of using only those intervals with relatively stable currents. This can be expected to introduce departures from prior analysis for two reasons. First, analysis that does not discriminate for stability will include periods during which the M-I system was dynamic, either because it transitioned between different imposed conditions or because of internal processes. Hence, some fraction of observations assigned to given solar wind/IMF parameters actually reflect dynamic behavior not 
specific to prolonged exposure to those conditions. A second reason is that the timing uncertainty in relating specific solar wind/IMF measurements to observations of the M-I system is comparable to the flight time of a low Earth orbiting satellite across the auroral zones, $\sim 25 \mathrm{~min}$. Advection estimates from L1 to Earth are uncertain to 10-20 min (e.g. Weimer et al., 2002). Moreover, the reconfiguration time of the M-I system is about 20 min (Murr and Hughes, 2001). Since the IMF is generally variable, even the best attempts to identify specific observations of the M-I system with particular solar wind/IMF measurements will assign the wrong driver estimate in some fraction of cases. Since we find that intervals exhibiting moderately stable currents for two hours happen only $5 \%$ of the time, unavoidable mis-assignment of values used to measure solar wind/IMF driving may be significant. By restricting analysis to intervals with stable currents, our analysis yields distributions that appear to more nearly reflect pure states of the M-I system.

For these reasons, even the best analysis of single satellite data would be subject to some mixing across bins in IMF direction, and the results will be subject to an admixture of M-I states. The southward IMF cases would be expected to contain observations that actually represent strong $B_{\mathrm{y}}$ or even northward IMF, and, hence, the apparent persistence of NBZ sense currents for southward IMF conditions could be expected. Conversely, observations tagged as northward IMF conditions could be expected to include strong $B_{\mathrm{y}}$ or southward IMF for which the Region-1 and 2 currents are strong leading to an over-estimate of the intensities of these currents.

The key factor enabling our results is the dramatic difference in the capabilities of data returned by Iridium and single satellites to specify the global state of the large-scale Birkeland currents. Consider how much data would be required from a single satellite to yield information on the large-scale currents roughly equivalent to that returned by Iridium. In one hour, the Iridium constellation returns independent data from six orbit tracks for identical solar illumination (UT, date, and EUV intensity) and solar wind/IMF driving conditions. Obtaining an equivalent set of observations from a single satellite would require six ideally distributed orbits for comparable solar illumination and solar wind/IMF conditions. The solar illumination (season, UT and perhaps F10.7) and solar wind/IMF conditions would need to be "matched" using statistical binning. One might use six season bins, twelve UT bins, three $E_{\mathrm{yz}}$ bins and eight IMF clock angle bins. Ignoring solar EUV intensity variations, this gives a total of $\sim 1700$ bins, comparable to the 1550 two-hour intervals employed here. For each of these bins one needs at least six orbits from different MLT planes, or nine hours of observations (90 min per orbit) corresponding to stable conditions. Since our analysis indicates that approximately $5 \%$ of the data would represent sufficiently stable conditions to allow equivalent stable current discrimination, one needs $180 \mathrm{~h}$ in each of the 1700 bins. Thus, a total of $\sim 300000 \mathrm{~h}, 12500$ days, or 34 years of observations would be needed to collect observations roughly comparable to those used in this study. Whether one could really get by with half as much observation time is almost immaterial; it is not feasible to extract equivalent information from single satellite observations. The Iridium data is therefore the key advance that we have leveraged here.

\section{Summary}

In this paper we present results from statistical analysis of Iridium-derived Birkeland currents to determine the dependence of the distribution morphology on IMF clock angle. The key results are summarized in Fig. 3. The current systems for southward IMF consist of the familiar Region 1 and 2 systems of Iijima and Potemra (1976) and the inferred flow associated with the most intense currents correspond to anti-sunward convection away from sub-solar magnetopause reconnection. As the IMF rotates duskward (positive IMF $B_{\mathrm{y}}$ ) the current distributions are distorted as the inferred outflow direction from magnetopause reconnection rotates from anti-sunward toward dawn in the Northern Hemisphere. The dawn-side downward Region 1 becomes contiguous across noon with dusk Region 2 while the upward Region 1 shrinks, rotates toward noon and moves poleward. As the IMF rotates northward with $B_{\mathrm{y}}$ positive, the currents with strongest intensity continue moving to the dayside with the upward current poleward of the downward current. This configuration corresponds to outflow from magnetopause reconnection from dusk to dawn in the Northern Hemisphere. For northward IMF, the inferred flow rotation is complete and the strongest upward current occurs on the dawn side, completing the reversal from the southward IMF Region 1 configuration, indicating sunward flow at highest latitudes corresponding to reverse convection from reconnection poleward of the cusp. The Region 2 currents are dramatically weaker than for southward IMF and are confined to the dayside poleward of $75^{\circ}$ MLAT. This evolution in the currents with IMF from south to north with positive $B_{\mathrm{y}}$ is mirrored with respect to the noon-midnight meridian for $B_{\mathrm{y}}$ negative. The results indicate that the M-I system achieves purer states than previous statistical analyses of Birkeland currents had indicated.

For southward IMF we find that the Region 1 and 2 current systems are not accompanied by statistically significant currents in the polar cap, poleward of $75^{\circ}$ MLAT. This is different from some previous statistical results which obtained persistent polar cap currents for southward IMF (e.g. Papitashvili et al., 2002). In this context one can appreciate the conundrum presented by a set of NBZ currents for southward IMF. If such a system were present in panel (S) of Fig. 3, one would need to impose a sunward flow at the pole toward noon. Since field lines threading the polar cap for southward IMF are generally accepted to be open, this would imply a secondary reverse dynamo on open field lines, and four-cell 
ionospheric convection for southward IMF. It is difficult to conceive of a configuration of reconnection or other mechanisms to produce a sustained vortical flow structure that could be active in this region. Simulations do not yield such structures. By showing that persistent NBZ-like currents are not present for southward IMF, the present results resolve a discrepancy between our observational knowledge of Birkeland currents and our understanding of M-I system dynamics for southward IMF.

The present results for northward IMF provide additional evidence that the viscous interaction is a secondary contributor to M-I driving. Discussion has continued for decades concerning the relative roles of reconnection of interplanetary magnetic field lines with the Earth's magnetic field on the one hand (Dungey, 1961), and viscous interaction between the solar wind flow and the magnetospheric circulation on the other (Axford and Hines, 1961), in driving the MI system. For northward IMF, reconnection occurs poleward of the cusp so that the two processes are spatially separated, and their relative contributions can be assessed by comparing the Birkeland currents associated with the plasma circulation they induce (Korth et al., 2005). The Weimer (2005) result found that for northward IMF the Region- 1 sense currents were of greater intensity than the filamentary NBZ currents nearest the pole. This would imply a significant contribution due to a viscous interaction vis-à-vis reconnection poleward of the cusp. In this study we find that the Region 2 currents are weak and narrowly confined for northward IMF and that the filamentary NBZ currents are more intense than the Region- 1 sense currents and carry essentially the same total current as the Region-1 sense currents. The total northward IMF Region 2 current is significantly less than $10 \%$ of the Region 2 total current for southward IMF implying that the viscous interaction plays a relatively minor role in solar wind-magnetosphere coupling. Simulation results of Sonnerup et al. (2001) for the magnetospheric response to interaction with an unmagnetized solar wind indicate a potential of almost $30 \mathrm{keV}$ for a nominal $400 \mathrm{~km} / \mathrm{s}$ solar wind speed. Our result suggests that the Sonnerup et al. (2001) result may be an over-estimate of the viscous contribution in the natural system either owing to numerical effects or to a reduction in the viscous interaction due to the magnetic field in the natural system. We note that Korth et al. (2005) found an upper limit for the viscous potential of $\sim 13 \mathrm{kV}$ consistent with the present statistical result of a roughly $10 \%$ contribution to southward IMF convection.

The results suggest that the Iridium stable currents data base should be further exploited. The data base could be used to determine the role that factors other than IMF clock angle have in governing the intensity of M-I solar wind coupling. This should yield new insight into the key factors governing the intensity of M-I solar wind coupling. Moreover, comparison of these results with simulation results will then allow us to assess how reliably the models capture not just the morphology of the coupling but also its intensity.
Acknowledgements. The efforts of Iridium Satellite LLC in providing engineering magnetometer data for scientific analysis are deeply appreciated. Support for processing and analysis of the Iridium magnetometer data was provided by NSF under grants ATM9819804 and ATM-0101064 and by NASA under grants NAG510849 and NAG5-12303. We thank the ACE team for the use of the MAG and SWEPAM data made available via the ACE Level 2 data base.

Topical Editor I. A. Daglis thanks N. Maynard and another anonymous referee for their help in evaluating this paper.

\section{References}

Anderson, B. J., Takahashi, K., and Toth, B. A.: Sensing global Birkeland currents with Iridium engineering magnetometer data, Geophys. Res. Lett., 27, 4045-4048, 2000.

Axford, W. I. and Hines, C. O.: A Unifying Theory of HighLatitude Geophysical Phenomena and Geomagnetic Storms, Can. J. Phys., 39, 1443-1464, 1961.

Cowley, S. W. H., Morelli, J. P., and Lockwood, M.: Dependence of Convective Flows and Particle Precipitation in the High-Latitude Dayside Ionosphere on the $\mathrm{X}$ and $\mathrm{Y}$ Components of the Interplanetary Magnetic Field, J. Geophys. Res., 96, 5557-5564, 1991.

Dungey, J. W.: Interplanetary Magnetic Field and the Auroral Zones, Phys. Rev. Lett., 6, 47-48, 1961.

Eriksson, S., Baker, J. B. H., Petrinec, S. M., Wang, M., Rich, F. J., Kuznetsova, M., Réme, M. W. D. H., Greenwald, R. A., Frey, H. U., Lühr, H., Ergun, R. E., Balogh, A., and Carlson, C. W.: On the generation of enhanced sunward convection and transpolar aurora in the high-latitude ionosphere by magnetic merging, J. Geophys. Res., 110, A11218, doi:10.1029/2005JA011149, 2005.

Haralick, R. M., Sternberg, S. R., and Zhuang, X.: Image Analysis Using Mathematical Morphology, IEEE Trans. Pattern Anal. Mach. Intell., PAMI-9, 532-550, 1987.

Heppner, J. P. and Maynard, N. C.: Empirical High-Latitude Electric Field Models, J. Geophys. Res., 92, 4467-4489, 1987.

Iijima, T. and Potemra, T. A.: The amplitude distribution of fieldaligned currents at northern high latitudes observed by Triad, J. Geophys. Res., 81, 2165-2174, 1976.

Iijima, T. and Potemra, T. A.: Large-Scale Characteristics of FieldAligned Currents Associated With Substorms, J. Geophys. Res., 83, 599-615, 1978.

Iijima, T., Potemra, T. A., Zanetti, L. J., and Bythrow, P. F.: LargeScale Birkeland Currents in the Dayside Polar Region During Strongly Northward IMF: A New Birkeland Current System, J. Geophys. Res., 89, 7441-7452, 1984.

Korth, H., Anderson, B. J., Wiltberger, M. J., Lyon, J. G., and Anderson, P. C.: Intercomparison of Ionospheric Electrodynamics From the Iridium Constellation With Global MHD Simulations, J. Geophys. Res., 109, A07 307, doi:10.1029/2004JA010428, 2004.

Korth, H., Anderson, B. J., Frey, H. U., and Waters, C. L.: HighLatitude Electromagnetic and Particle Energy Flux During an Event With Sustained Strongly Northward IMF, Ann. Geophys., 23, 1295-1310, 2005, http://www.ann-geophys.net/23/1295/2005/. 
McComas, D. J., Bame, S. J., Barker, P. L., Feldman, W. C., Phillips, J. L., Riley, P., and Griffee, J. W.: Solar Wind Electron Proton Alpha Monitor (SWEPAM) for the Advanced Composition Explorer, Space Sci. Rev., 86, 563-612, 1998.

Merkin, V. G., Lyon, J. G., Anderson, B. J., Korth, H., Goodrich, C. C., and Papadopoulos, K.: A global MHD simulation of an event with a quasi-steady northward IMF component, Ann. Geophys., 25, 1345-1358, 2007, http://www.ann-geophys.net/25/1345/2007/.

Murr, D. L. and Hughes, W. J.: Reconfiguration timescales of ionospheric convection, Geophys. Res. Lett., 28, 2145-2148, 2001.

Ohtani, S., Potemra, T. A., Newell, P. T., Zanetti, L. J., Iijima, T., Watanabe, M., Blomberg, L. G., Elphinstone, R. D., Murphree, J. S., Yamauchi, M., and Woch, J. G.: Four large-scale fieldaligned current systems in the dayside high-latitude region, J. Geophys. Res., 100, 137-153, 1995.

Papitashvili, V. O. and Rich, F. J.: High-latitude ionospheric convection models derived from Defense Meteorological Satellite Program ion drift observations and parameterized by the interplanetary magnetic field strength and direction, J. Geophys. Res., 107, SIA17-1-13, 2002.

Papitashvili, V. O., Christiansen, F., and Neubert, T.: A new model of field-aligned currents derived from high-precision satellite magnetic field data, Geophys. Res. Lett., 29, 1683, doi:10.1029/ 2001GL014207, 2002.

Potemra, T. A., Zanetti, L. J., Bythrow, P. F., Lui, A. T. Y., and Iijima, T.: $B_{\mathrm{y}}$-Dependent Convection Patterns During Northward IMF, J. Geophys. Res., 89, 9753-9760, 1984.

Ruohoniemi, J. M. and Greenwald, R. A.: Statistical patterns of high-latitude convection obtained from Goose Bay HF radar observations, J. Geophys. Res., 101, 21 743-21 763, 1996.

Ruohoniemi, J. M. and Greenwald, R. A.: Dependencies of high-latitude plasma convection: Consideration of interplanetary magnetic field, seasonal, and universal time factors in statistical patterns, J. Geophys. Res., 110, A09 204, doi:10.1029/ 2004JA010815, 2005.

Smith, C. W., L'Heureux, J., Ness, N. F., Acuña, M. H., Burlaga, L. F., and Scheifele, J.: The ACE Magnetic Fields Experiment, Space Sci. Rev., 86, 613-632, 1998.
Sonnerup, B. U. O., Siebert, K. D., White, W. W., Weimer, D. R., Maynard, N. C., Schoendorf, J. A., Wilson, G. R., Siscoe, G. L., and Erickson, G. M.: Simulations of the magnetosphere for zero interplanetary magnetic field: The ground state, J. Geophys. Res., 106, 29419-29434, 2001.

Waters, C. L., Anderson, B. J., and Liou, K.: Estimation of global field aligned currents using the Iridium System magnetometer data, Geophys. Res. Lett., 28, 2165-2168, 2001.

Waters, C. L., Anderson, B. J., Greenwald, R. A., Barnes, R. J., and Ruohoniemi, J. M.: High Latitude Poynting Flux From Combined Iridium and SuperDARN Data, Ann. Geophys., 22, 28612875, 2004, http://www.ann-geophys.net/22/2861/2004/.

Weimer, D. R.: Models of High-Latitude Electric Potentials Derived with a Least Error Fit of Spherical Harmonic Coefficients, J. Geophys. Res., 100, 19595-19607, 1995.

Weimer, D. R.: A flexible, IMF dependent model of high-latitude electric potentials having "space weather" applications, Geophys. Res. Lett., 23, 2549-2552, 1996.

Weimer, D. R.: Maps of ionospheric field-aligned currents as a function of the interplanetary magnetic field derived from Dynamics Explorer 2 data, J. Geophys. Res., 106, 12 889-12902, 2001.

Weimer, D. R.: Improved ionospheric electrodynamic models and application to calculating Joule heating rates, J. Geophys. Res., 110, A05 306, doi:10.1029/2004JA010884, 2005.

Weimer, D. R., Ober, D. M., Maynard, N. C., Burke, W. J., Collier, M. R., McComas, D. J., Ness, N. F., and Smith, C. W.: Variable time delays in the propagation of the interplanetary magnetic field, J. Geophys. Res., 107, 1210, doi:10.1029/2001JA009102, 2002.

Zanetti, L. J., Potemra, T. A., Iijima, T., Baumjohann, W., and Bythrow, P. F.: Ionospheric and Birkeland Current Distributions for Northward Interplanetary Magnetic Field: Inferred Polar Convection, J. Geophys. Res., 89, 7453-7458, 1984. 